OPEN

SUBJECT AREAS:

PERCEPTION

HUMAN BEHAVIOUR

Received

19 May 2014

Accepted

28 July 2014

Published

3 September 2014

Correspondence and requests for materials should be addressed to V.V.P. (v.van. polanen@vu.nl)

\section{Target contact and exploration strategies in haptic search}

\author{
Vonne van Polanen, Wouter M. Bergmann Tiest \& Astrid M. L. Kappers
}

MOVE Research Institute, Faculty of Human Movement Sciences, VU University, Amsterdam, The Netherlands.

In a haptic search task, one has to detect the presence of a target among distractors using the sense of touch. A salient target can be detected faster than a non-salient target. However, little is known about the exploration strategies that are used, especially in 3D search tasks where items are held in the hand. In this study, we investigated which parts of the hand were used to contact the target and which strategies were performed. Blindfolded participants performed search tasks in four conditions, where the targets differed in relevant property and saliency. The positions of the target and the hand were tracked during exploration. It was found that target saliency had a large effect on the use of the hand parts and the strategies. In the non-salient target conditions, the fingers, especially the thumb, contacted the target more often than in the salient target conditions. This could also be seen in the strategies, where the thumb was used to explore the items in a serial way by moving them in the hand or touching them individually. In the salient target conditions, more parallel strategies like grasping or shuffling of the items in the hand were used.

umans are very skilled in exploring multiple objects at the same time by touch. With a handful of objects, a desired object can usually be found quite well among the others. The search for your keys in your bag, or for a specific button on a control panel, are both examples of haptic search tasks. In a search task, one searches for a desired object (the target) among other objects that are not of interest (the distractors). Some features can be detected immediately among other items that do not possess that feature. This is called the pop-out effect ${ }^{1}$. The feature that is present in the target is then said to be salient with respect to the distractor property and to be processed very efficiently. In vision research, search tasks have been widely used to investigate the processing of different features. Recently, there has also been a growing interest in the search capabilities and efficiency using the haptic modality ${ }^{2-7}$.

When searching for a target, many different search strategies can be used. Usually, a division is made between parallel strategies (explore items all at once) and serial strategies (explore items one at a time). This is often based on the reaction time, which increases with the number of items in a serial search, giving a positive search slope. With a parallel strategy, the search will be independent of the number of items and thus give a flat search slope. In visual search, a variation in eye movements can also be seen ${ }^{8}$, but in haptic search, with the many degrees of freedom in the hand, the number of possible strategies might be much larger.

In $2 \mathrm{D}$ haptic search tasks, where the items are placed on a display, some investigation of the performed explorations has been done. Differences in velocity profiles ${ }^{9}$, normal force ${ }^{10}$, speed and travelled distances ${ }^{3,6}$ were $^{2}$ found between tasks that differed in search difficulty. When trials were classified into movement types according to quantitative measures, it was shown that more simple strategies were used with a salient target and more detailed strategies in a more difficult search ${ }^{11}$.

In contrast to these $2 \mathrm{D}$ hand analysis, the detailed analysis of movement strategies in $3 \mathrm{D}$ haptic search tasks has yet to be performed. In previous studies, where handheld items were searched, only the percentage of trials in which items that are dropped out of the hand were calculated ${ }^{4,7,12}$, but this gave only limited information about the strategies that were used. Until now, there has been little attempt to quantify these strategies in the search for handheld objects.

In fact, the manipulation of multiple items in the hand is a topic that has been rarely studied. In the context of haptic exploration of single objects, movements have been analysed using video analysis ${ }^{13}$ or quantitative measures $^{14}$. Haptic perception is an active process, so also when multiple objects are present in the hand they need to be moved through the hand to perceive most of their properties. With multiple items, the exploration movements might also depend on the perceived property, but other issues like keeping track of which item is felt and the handling of items that occlude each other must be handled as well.

Another interesting aspect of haptic search is that the hand that is used to perform this task contains many different sensors that are spread unevenly over parts of the hand. For instance, the fingertips are more densely 
populated than the palm of the hand ${ }^{15}$, making them more sensitive to small differences in properties. This does not mean that in a haptic search task the hand palm would not contribute to the perception of a target. An interesting question, then, is which parts of the hand contribute to the search. One can imagine that for more difficult searches, the fingers are used more extensively to explore the items than in easy searches.

In the present study, we had two aims in analysing the exploratory movements that are used in a haptic search task. First, we were interested in which parts of the hand were used to perceive the target. The second objective was to analyse which strategies are used to perform the task. We investigated four search conditions: two in which the target differed from the distractors in shape (spheres and cubes) and two in which it differed in texture (rough and smooth). In either group there was a difficult search (sphere among cubes or smooth among rough) and an easy search (cube among spheres or rough among smooth). The easy search tasks were for salient targets and the difficult search tasks were for non-salient targets, as known from previous research ${ }^{3,4,12}$.

In each of the four conditions, participants had to quickly and accurately determine whether a target was present or not. The time it took until they gave a response was the reaction time. Furthermore, the hand movements were measured by taping sensors to the hand. From these data, a model of the hand was made ${ }^{16}$. With this model, a contact analysis was performed to determine which parts of the hand contacted the target at what time.

The second analysis was the investigation of the movement strategies that were used in the search. Four strategies were defined and each trial was classified by a set of variables into one of those four. The strategies were named shuffle, grasp, manipulate and thumb and could be described as follows:

- Shuffle was a movement in which the items were shuffled in the hand. They were not felt individually, but quickly moved within the hand.

- Grasp was a grasp of the stimuli, in which no fine movements were made. This was usually a short movement, in which the answer was given immediately.

- Manipulate was a movement in which the items were moved individually with the thumb in the hand, more or less one by one, and sometimes dropped out of the hand.

- Thumb was a strategy where the items laid in the outstretched hand and the thumb individually touched the items, one by one.

These strategies were based on the observation of the trials and that of previous studies. They were chosen because they were regularly observed and were also distinct enough to allow the trials to be grouped into different categories. Therefore, the four strategies should be seen as different classes, where a variation of movements is possible but still belonging to the same group.

\section{Results}

Since the analysis of the contact with the target yielded many significant results, this section will only focus on the most prominent results. The full report of the results can be found in the Supplementary Information.

Reaction times and errors. The reaction times and errors are presented in Table 1. As can be seen, lower reaction times and error percentages were found in the search for a cube and a rough target than in the smooth and sphere conditions.

Contact with the target. The segments of the hand that were analysed were the hand palm, and three phalanges (distal, middle and proximal) of each finger, including the thumb. The percentage of trials in which a segment was in contact with the target at any moment in the trial is displayed in Fig. 1 . In 5 trials $(0.5 \%)$ no
Table 1 The reaction times and percentage of errors in each condition, shown for target-present and target-absent trials separately. Reaction times are means \pm standard errors of the mean. Error percentages are calculated as the number of errors divided by the number of trials + the number of errors*

\begin{tabular}{lcccc} 
& \multicolumn{2}{c}{ Reaction times (s) } & \multicolumn{2}{c}{ Errors (\%) } \\
\cline { 2 - 5 } & present & absent & present & absent \\
\hline cube & $1.12 \pm 0.13$ & $2.36 \pm 0.30$ & 1 & 0 \\
sphere & $2.28 \pm 0.15$ & $6.11 \pm 0.55$ & 8 & 0 \\
rough & $1.26 \pm 0.09$ & $2.54 \pm 0.19$ & 3 & 0 \\
smooth & $3.68 \pm 0.38$ & $8.54 \pm 0.86$ & 12 & 0 \\
\hline
\end{tabular}

*Percentages were rounded to integers.

contact was found with any hand part during the trial. This was only in the conditions with a rough target or a cubical target.

It was not desirable to conduct one Analysis of Variance (ANOVA) on all the segments, since the hand palm segment did not consist of different phalanges whereas the different fingers do. Treating each segment as a separate factor would have made the interpretation quite difficult as well. Therefore, we decided to compare the four segment groups (distal, middle and proximal phalanx and hand palm) with each other and subsequently analyse each segment group separately. The ANOVA values can be found in the Supplementary Information, in Tables S1-3.

As can be seen in Fig. 1, it is clear that the percentage of target contacts differs between the different parts of the hand. A 4 (segment group) $\times 4$ (condition) repeated measures ANOVA indicated that the hand palm and distal segment had the most contact with the target.

To evaluate the differences between the fingers, the distal, middle and proximal segments were evaluated separately. The target contact with the distal segment was further examined in a 5 (finger) $\times 4$ (condition) repeated measures ANOVA. Effects of finger, condition, and an interaction effect of finger $\times$ condition were found. The index finger and the little finger seemed to make less contact with the target compared to the other fingers. Differences between the fingers were more prominent in the sphere and smooth conditions. The main effect of condition in the distal phalanx indicated that the target was touched less often in the cube and rough conditions than in the sphere and smooth conditions. The interaction effect of condition $\times$ finger indicated that the conditions differed primarily in the use of the thumb, and also in the index and little finger. In Fig. 1, these differences between the conditions in the amount of target contact with the thumb can be clearly seen.

The analysis of contact with the middle phalanx from a 5 (finger) $\times 4$ (condition) repeated measures ANOVA revealed effects of finger, condition, and an interaction effect of finger $\times$ condition. The middle and ring finger were contacted more than other fingers, but differences were only significant in the sphere and smooth conditions. Similar condition differences as for the distal phalanx were found, but primarily in the little finger and also in the middle finger.

For the proximal phalanx, a 5 (finger) $\times 4$ (condition) repeated measures ANOVA was performed. An effect of finger was found. Post-hoc tests revealed that the thumb less frequently touched a target than the index, middle and ring finger. In addition, less contact was observed for the little finger compared to the middle and ring finger. There was no effect of condition or an interaction effect. A repeated measures ANOVA on the hand palm contact data showed no significant effect of condition.

In sum, the hand palm and the distal segment most often made contact with a target. Differences between the conditions seemed to exist mainly in the distal and middle segments, with more contact in the sphere and smooth conditions than the rough and cube condi- 
cube
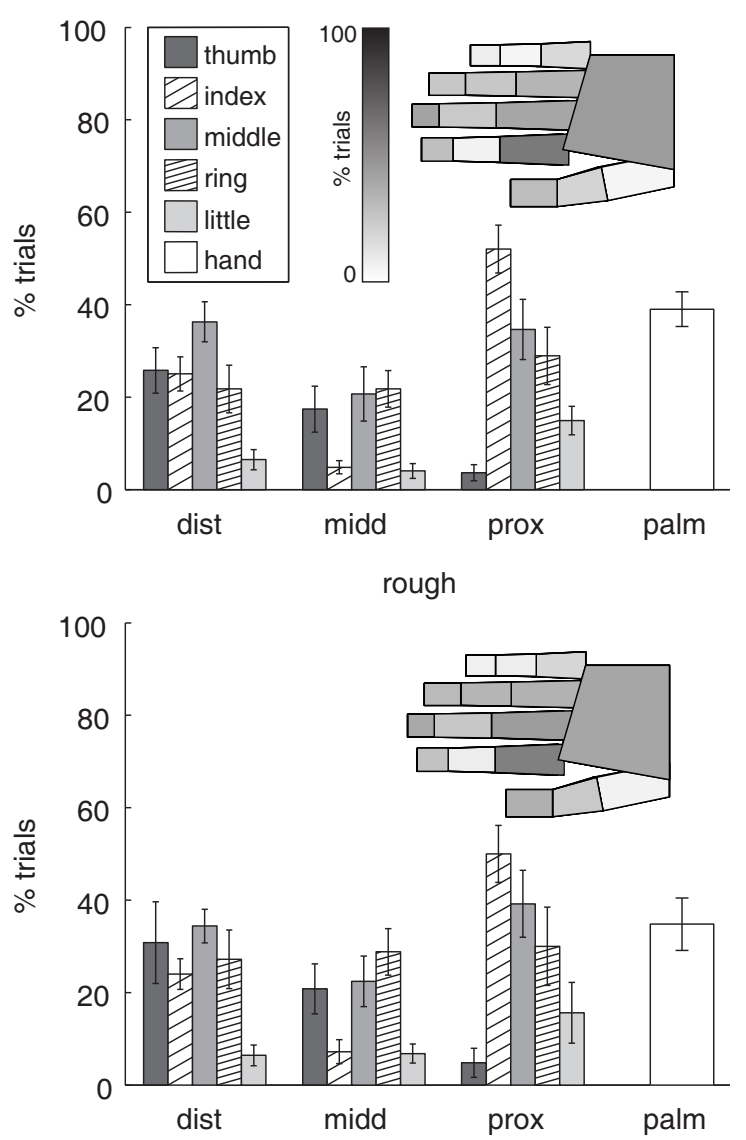

sphere
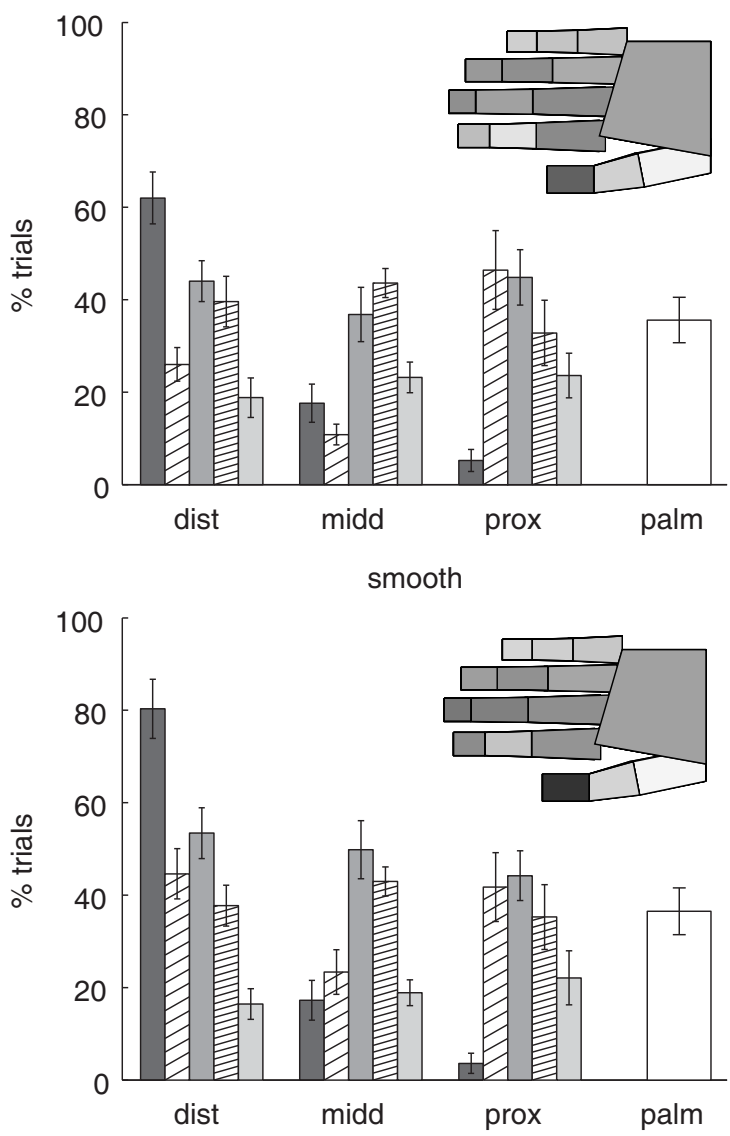

Figure $1 \mid$ The percentage of trials in which a segment was in contact with the target in the trial. Each panel (condition) shows data for the distal (dist), middle (midd) and proximal (prox) segments, for each finger (thumb, index finger, middle finger, ring finger and little finger), and for the hand palm segment. Error bars indicate standard errors. The top right of each panel shows the data plotted on a hand model. Darker colours indicate a higher percentage of trials with contact.

tions. For the distal segment, the conditions differed primarily in the use of the thumb, whereas they differed most in the little finger for the middle segment. Most differences between the fingers were found in the smooth and sphere conditions.

The amount of contact might also differ over the course of the trial. To investigate this, the target contact was analysed at different moments in time. Each trial was divided into 20 intervals, to examine changes over the course of the trial. When the contact points with the targets were inspected at different time intervals, no clear patterns were seen in the middle and proximal segments or the hand palm segment. In these segments, the amount of contact did not differ much over time. In the distal segment, differences in time were found, as can be seen in Fig. 2. At the end of the trial, the percentage of trials in which a target was contacted seemed to increase rapidly, primarily for the thumb in the smooth and sphere conditions.

A 5 (finger) $\times 4$ (condition) $\times 2$ (time step) repeated measures ANOVA was performed on the data of the distal segment. The first and last interval were chosen as time steps to examine the differences at the start and end of the trial. All main effects and interactions were significant. The finger and condition effect replicated results described above and can be found in the Supplementary Information. The effect of time step indicated that more finger contact was seen at the end of the trial than at the beginning. This increase in amount of contact with time was significant for the index finger in the rough, sphere and smooth conditions. In the cube condition, an increase was found for the middle finger and thumb. As can be clearly seen in Fig. 2, this increase in contact was also significant for contact with the thumb in the sphere and smooth condition.
In the analysis, even a contact point at a single sample counted as a touch of the target. One sample corresponds to $10 \mathrm{~ms}$, so this might not reflect an actual manipulation of the target. Therefore, an analysis was performed where the contact points were checked in a window of $100 \mathrm{~ms}$. If in at least $70 \%$ of the samples in a window the target was contacted, this was seen as a long contact period. If only these longer contact periods were used in the analysis, similar results were found as described above. Overall, the percentage values were somewhat lower, but the patterns remained the same.

Movement classification. The second aim of the study was to examine the exploration strategies. Trials were classified into the four different strategies as described in the Introduction. Examples of the strategies are illustrated in Fig. 3 to give some indication of the exploratory motions. These are movie stills from videos that can be found in the Supplementary Information. A set of criteria were chosen to distinguish between the strategies. The criteria variables were the speed of the thumb and the ring finger, the volume in which the thumb moved and the bending and stretching of the ring finger. The expected patterns are indicated in Table 2. The results are shown in Fig. 4 . The grasp strategy was used most often, especially when the target was present. For the cube and rough conditions, this strategy was used primarily, whereas in the sphere and smooth conditions different kinds of strategies were also frequently seen. When the target was absent, larger differences between the conditions were found. In the sphere and smooth conditions, most often the manipulate strategy was used, whereas most trials in the rough and cube conditions showed grasp strategies. 
cube

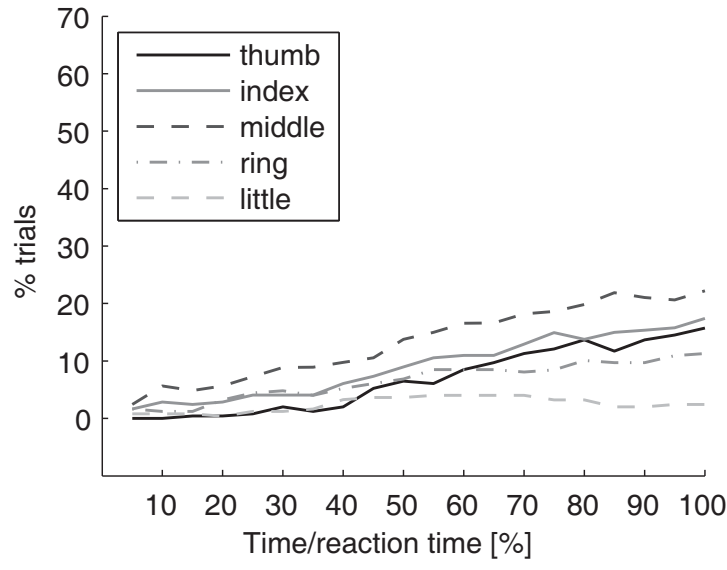

rough

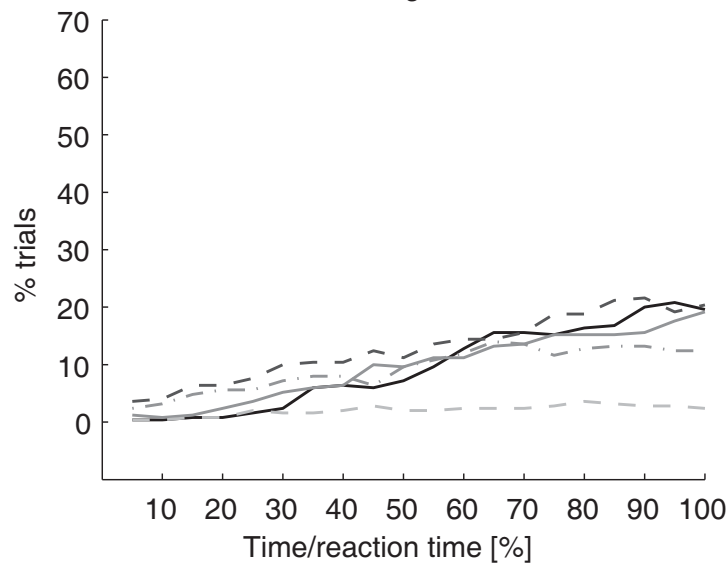

sphere

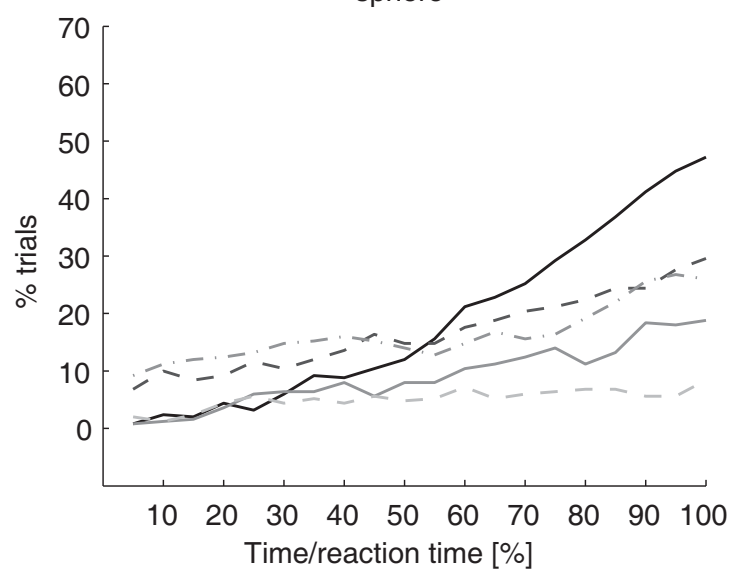

smooth

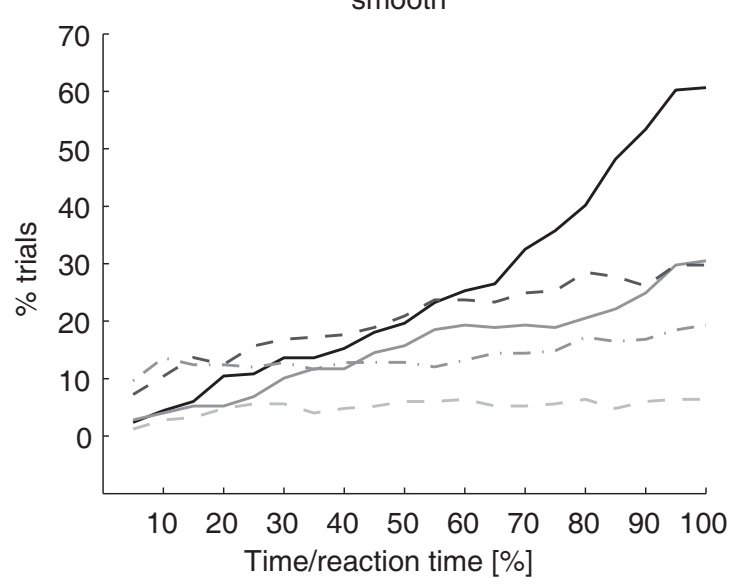

Figure $2 \mid$ The percentage of trials in which the distal segment was in contact with the target, for each $5 \%$ interval of the trial. Lines represent the different fingers.

\section{Discussion}

In this study we investigated the explorations that are used in a haptic search task. Specifically, we focused on two aspects. The first aim was to study which parts of the hands are used. Secondly, the strategies that are used in the explorations were of interest. We investigated these aspects in four search tasks. Two properties, shape and texture, were used to distinguish the target from the distractors.

Previous research has shown that edges and vertices (present in cubes) and roughness are salient features ${ }^{2-4,12}$. Similar to those studies, we found shorter reaction times and a smaller number of errors in the search for cube among spheres or a rough sphere among smooth spheres than in the search for the reversed situations. These results confirmed, as a baseline, that the searches for cubes and rough spheres were easier than the searches for spheres and smooth spheres. This was used to further investigate the exploration movements that were used in these search tasks.

For the first objective of this study, we examined which parts of the hand were used in the search for a target. Therefore, we checked which parts of the hand were in contact with the target during the exploration. There were some general observations with respect to target contact that did not depend on the kind of target. Results indicated that especially the hand palm and the fingertips were used to perceive the target. The middle and proximal phalanges made less contact with the target. This is probably because all the items are held in the hand with bent fingers. The middle and proximal phalanges are folded together and thus do not contact the target often.

The differences in the amount of target contact between the search for a shape or a texture feature did not appear to be very large. The
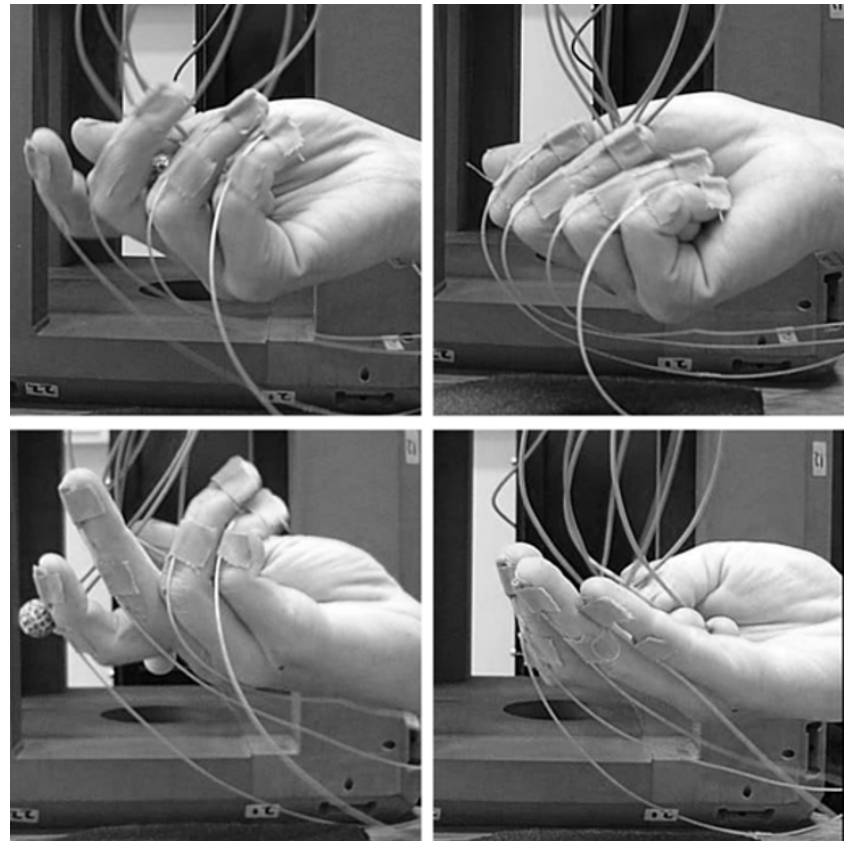

Figure 3 | Movie stills of four examples of the movement strategies. From top left: shuffle, grasp, manipulate, thumb. The movies with the corresponding hand model can be found in the Supplementary Information. 


\begin{tabular}{|lcccc|}
\hline \multicolumn{4}{|l|}{ Table 2 | Variables used to classify the strategies } \\
variable & shuffle & grasp & manipulate & thumb \\
\hline speed thumb & high & low & high & high \\
speed ring finger & high & low & high & low \\
volume thumb & low & low & high & high \\
turning points & high & low & high & low \\
\hline
\end{tabular}

saliency of the target seemed to account for most of the differences between the search conditions: if the target was not salient, as in the sphere and smooth conditions, more target contact was seen than in the conditions with a salient target (rough and cube). The differences between the conditions were only found in the use of the distal and middle parts of the fingers. The difference in the percentage of thumb contact between the conditions was especially large. The higher amount of contact indicates that the fingers were used more often to perceive the target in these conditions. This is a reasonable result, because the fingers are more densely innervated with tactile receptors than other parts of the hand ${ }^{15}$ and are thus more sensitive. Indeed, it might be that only once the fingers touch the target it is identified, whereas for an easily perceivable target, contact with less sensitive parts of the hand might already be enough.

This important role of the fingertips was also seen when looking at the moment of contact. At the end of the trial, the thumb was more often contacted than at the beginning in the conditions with a nonsalient target. Such behaviour suggests that once the thumb touches the target, it is identified and a response can be given. In the current task, with handheld items, the thumb can easily move over and touch an item to identify it.

A few issues must be noted when interpreting the contact with the target. With the longer trials, as in the difficult tasks, the chance to accidentally touch the target with a certain hand part is higher. However, similar results were found with longer contact durations. In addition, clear strategy differences were seen between the tasks, which indicate a change in how the fingers are used. Therefore, we believe the contact differences are not due to chance. Secondly, it must be noted that the fact that the participant touched the target does not mean that this contact is consciously perceived, for instance, due to light touches or attentional focus. This makes it difficult to relate the contact to the participants' response. However, if a target is touched more often with a specific hand part, it is likely that it is also consciously perceived more often with that hand part. Therefore, we think that our results do reflect how the separate hand parts are used and that these parts are probably even necessary to perform the task.

The second analysis investigated the strategies that were used in the search tasks. Similar to the use of the various hand parts, no clear differences were seen between the search for a texture or shape feature in the exploration strategies. This might seem surprising, because very different exploratory procedures are optimal for these properties, i.e., enclosure for shape and lateral motion for textures ${ }^{13}$, although these exploratory procedures were defined for larger objects. Possibly, the roughness of the rough target was so obvious it could be felt with just a small movement, not clearly visible as a different strategy. In addition, the choice of the strategy categories might not be sensitive enough for the differentiation between searches for the two property dimensions.

Nevertheless, the movement classification showed clear strategy differences between the different tasks with respect to the target saliency. In searching for a cube or a rough sphere among smooth spheres, a single grasp was often enough to detect the presence of the target and the grasp strategy was used most. In searching for a nonsalient target, this strategy was less often used, and especially when the target was absent, more extensive and serial explorations were necessary, as seen in the frequent use of the manipulate strategy in these conditions. This difference of simple explorations in easy searches compared to more detailed explorations in difficult searches was also seen in previous search studies that used 2D set-ups ${ }^{3,6,11}$. Participants did not know whether a target was present or not and probably did not adjust their strategy much to the presence of the target, but they could stop their exploration once the target was felt. If a detailed exploration was intended, but the target was felt early in the search, the exploration could be aborted in a target-present trial. In a target-absent trial, the movements would have continued. Although the trials would be classified differently, the intention might have been the same.

These results confirm the assumption that parallel strategies can be used if the target is salient, but not when it is difficult to detect. This is the first time this is shown in a quantitative movement analysis in 3D search tasks. Previous research mainly used search slopes to distinguish between parallel and serial strategies. Our results indicate that these commonly found differences in search slopes are indeed caused by the performed strategies. Interestingly, these clear differences between search tasks can already be made apparent by studying a single number of items. In the tasks with a salient target, a rough or cubical target, a grasp or a shuffling movement was almost always used. Both of these exploration patterns can be considered parallel strategies. In the search for a smooth or spherical target, detailed serial strategies are performed, in which the thumb is used to individually search the items.

The movement classes were chosen based on previous research and observation. Of course, many more strategies could be used and intermediate behaviours were also seen. This also emphasises that parallel and serial strategies can exist in many forms. The four strategies reflect some of the possible exploration categories that are used in search tasks and the criterion variables were chosen to distinguish between these categories. Overall, the differences between the conditions are large enough to give an insight into the influence of target saliency on the exploration strategies in haptic search. Future

\begin{tabular}{c|c|c|c|c|}
\multicolumn{1}{c}{} & \multicolumn{4}{c}{ target present } \\
\cline { 2 - 5 } shuffle & \multicolumn{3}{c}{ grasp manipulate thumb } \\
\cline { 2 - 5 } cube & 24 & 73 & 0 & 3 \\
\cline { 2 - 5 } sphere & 16 & 59 & 16 & 10 \\
\cline { 2 - 5 } rough & 15 & 82 & 2 & 1 \\
\cline { 2 - 5 } smooth & 8 & 51 & 25 & 16 \\
\cline { 2 - 5 } & & &
\end{tabular}

\begin{tabular}{c|c|c|c|c|}
\multicolumn{1}{c}{} & \multicolumn{4}{c}{ target absent } \\
\cline { 2 - 5 } shuffle & \multicolumn{3}{c}{ grasp manipulate thumb } \\
\cline { 2 - 5 } & 27 & 70 & 2 & 2 \\
\cline { 2 - 5 } sphere & 18 & 6 & 70 & 7 \\
\cline { 2 - 5 } rough & 21 & 76 & 2 & 1 \\
\cline { 2 - 5 } smooth & 7 & 2 & 84 & 6 \\
\cline { 2 - 5 } & & & &
\end{tabular}

Figure $4 \mid$ Percentage of trials of the classified strategies in each condition, for target-present and target-absent trials separately. Numbers are sized proportionally to their values, so greater sizes are used for higher percentages. 
research could aim to improve the classification. A useful addition would be to look at the strategy usage over time, so switches could be made apparent. The current criteria are not suitable for such an analysis, but serve as a first step in the classification of search behaviour. Another topic for future studies might be to look at the strategies used for the localisation of objects, which is also important in daily life searches.

The kind of strategy that is used influences which parts of the hand contact the target. For example, in a grasp strategy, the fingertips will not touch the target in most cases. This strategy was most often used in the conditions with a salient target, a cube or a rough sphere, and also the amount of target contact was low in these conditions. On the other hand, which parts of the hands need to be used can also determine the strategy choice. It seems that the more sensitive parts of the hand, i.e., the fingertips, need to be used to detect the presence of a non-salient target. To use the fingertips to touch the target, the items need to be shuffled through the hand. In the case of a difficult target, the items might need to be searched in a serial manner. To keep track of which item is felt, they must be explored in a coordinated way. For instance, they might be dropped out of the hand one by one, or felt individually in the hand. These strategies were seen more often in the conditions with non-salient targets, the smooth spheres among cubes or rough spheres. In these conditions, the thumb was used often to contact the target. The thumb has a wide range of motion and can be easily used to move items through the hand or explore items that are arranged on the hand palm. Our study investigated in-hand manipulation. In other tasks where, for instance, the hand explores objects more from above instead of grasping, slightly different strategies and contacts might be seen. However, we expect the difference between a parallel or serial strategy and increased contact for difficult tasks compared to easy tasks to remain.

In conclusion, humans seem to be very skilled to adjust their movements to the specific task, also in the exploration of multiple objects. In a haptic 3D search task the use of hand parts for exploration of the stimuli depends on the saliency of the target. This is the first study that has investigated the exploration behaviour of a $3 \mathrm{D}$ haptic search task in detail and in a quantitative manner. This information can be useful in the development of tactile sensors for robots or haptic devices. Although many different sensors exist, still much can be learned from human data to improve robotic designs ${ }^{17,18}$.The placement and sensitivity of the sensors as well as the movement strategies could be adjusted to the specific task that is performed with such a device.

\section{Methods}

Additional information about the apparatus, methods and analysis is described in the Supplementary Information.

Participants. Ten participants ( 6 females, $22 \pm 3$ years old) took part in the experiment. All participants were right-handed according to Coren's test ${ }^{19}$ and used their right hand for performing the experiment. Participants signed an informed consent form prior to the experiment. This study was conducted in accordance with principles as stated in the declaration of Helsinki and was approved by the Ethics Committee of the Faculty of Human Movement Sciences of the VU University, Amsterdam.

Apparatus. The hand movements were measured with a 3D Guidance TrakSTAR movement tracking system (Ascension Technology Corporation), that measured 6 degrees of freedom. One sensor was placed on the nail of each of the five fingers, including the thumb, and one sensor was placed on the back of the hand, approximately in the middle in line with the knuckle of the middle finger. Two sensors were placed inside the stimuli (see below).

Three types of wooden stimuli were used in the experiment: rough spheres, smooth spheres and smooth cubes (Fig. 5). The rough spheres were created by gluing small pieces of sandpaper (Bosch, P60) on the spheres ${ }^{12}$. A stimulus set for a single trial always consisted of seven items. This number was chosen because this was the largest number that could still fit comfortably in the hand and greater strategy differences between the conditions can be expected with a larger number of items.

Two stimuli were attached to the sensors and the other five stimuli were attached to dummy wires, made from rubber tubes. The sensor wires were coated with the same material as the dummy wires, so all wires felt the same. In trials with no target present,

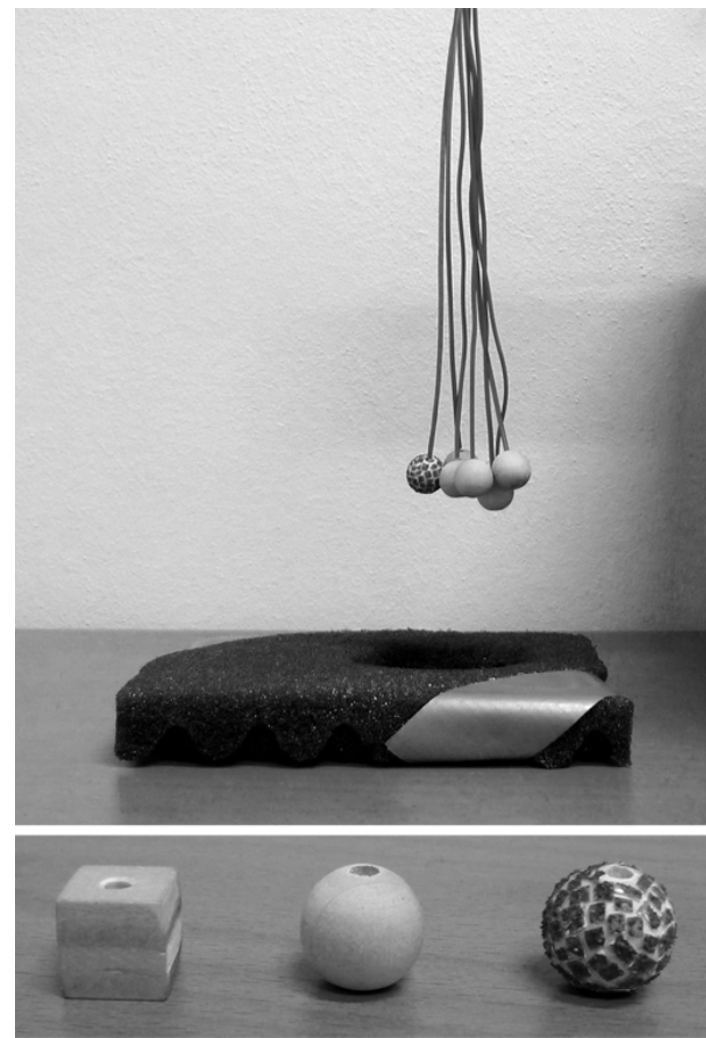

Figure 5 The experimental set-up. Top: the stimuli hang from wires above a resting cushion. Below: the stimuli; a smooth cube, a smooth sphere and a rough sphere.

two distractors contained a sensor. When the target was present, one sensor was placed in the target and one in a distractor.

Procedure. There were four conditions: a smooth cube among smooth spheres (cube), a smooth sphere among smooth cubes (sphere), a rough sphere among smooth spheres (rough) and a smooth sphere among rough spheres (smooth). In each condition, the procedure was similar. Participants had to determine whether a target was present or not. The identity of the target and the distractors was explained before the start of a condition. When the measurement was started, they lifted their hand to initially grasp the bunch of stimuli, which started the reaction time measurement. After that, they were free to manipulate the stimuli in any way they preferred. However, they had to be as fast as possible, but also make as few mistakes as possible. They indicated their answer by calling out 'yes' or 'no'. Their response, as recorded with the microphone of a head-set that was placed on the participants' head, stopped the measurement. They received feedback about the correctness of their answer. Erroneous trials were repeated at the end of the condition. Participants were blindfolded during the experiment. Preceding a condition, participants performed a practice block. 25 target-present trials and 25 target-absent trials were presented in a randomised order. The order of the conditions was randomised among participants.

Hand model. To be able to evaluate which parts of the hands were used in the search and to investigate the movement strategies, a model of the hand was used ${ }^{16}$. The hand was modelled as a number of connected segments, where the hand palm consisted of one rigid body and the fingers and thumb had 3 segments each; one for each phalanx (distal, middle and proximal). The joint positions were calculated from the sensor positions and the segments were constructed around these joint parts.

A detailed description of the calculations to construct the hand model can be found elsewhere ${ }^{16}$. The joint positions for the distal joint (near the fingertip) were calculated by transforming the nail sensor coordinates. The knuckle joint was determined by the transformation of the coordinates of the sensor on the back of the hand. The proximal joint (between middle and proximal phalanx) was determined by solving a set of equations. Here, a triangle was assumed between the distal joint, the proximal joint and the knuckle joint.

The segments were constructed by transforming the sensor coordinates or by summations of vectors around the calculated joint coordinates. In this way, each segment was formed as a rigid block with 8 vertices. An example of the modelled hand is shown in Fig. 6.

The constant measures of the hand (length, width and thickness of the segments) and the initial orientation of the sensors were determined from a photograph of the hand and a calibration procedure ${ }^{16}$. 


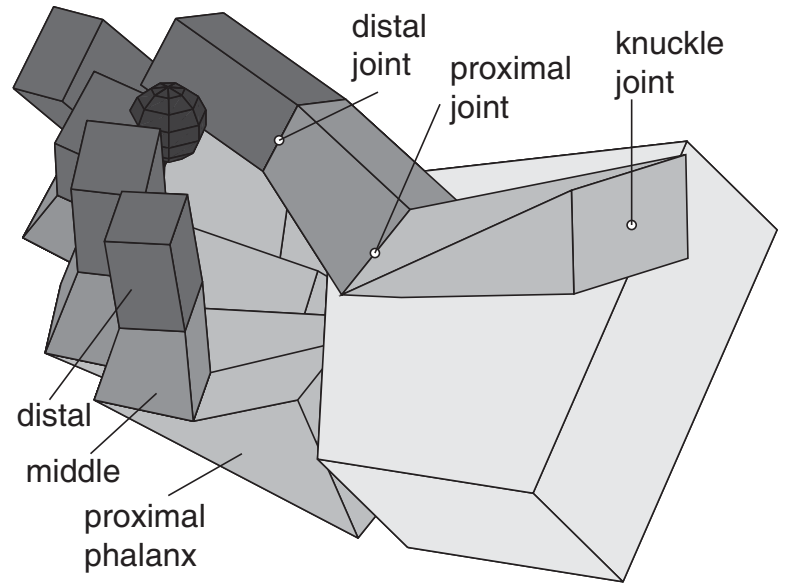

Figure $6 \mid$ An example of the modelled hand including a target (black sphere). For the little finger, the distal, middle and proximal phalanx are specified. For the thumb, the distal, proximal and knuckle joint are indicated. The distal and proximal joints were located inside the fingers, but are drawn on top for illustration purposes.

Analysis. The time between the start (touch of stimuli) and end of the trial (vocal response) was the reaction time. Only correctly answered trials were included in the analysis. Two analyses were performed for the investigation of the hand motions. In the first analysis, the contact with the target of different hand parts was evaluated. Here, only target-present trials were used, since only these trials contained a target. In the second analysis, the movement strategies that were used for exploration were investigated. This analysis included both target-present and target-absent trials.

Analysis of target contact. In this analysis, we calculated which parts of the hand were in contact with the target during exploration. The model of the hand was used to determine the location of all parts of the hand. To see where the target touched the hand during the trial, the distance of the target to the 6 planes of each segment (five distal phalanges, five middle phalanges, five proximal phalanges and the palm of the hand) was calculated. If the distance to one or more of the planes of a segment was equal or less than the target radius, this was counted as a contact point with this segment. The target was always assumed to be spherical with a radius of $7.5 \mathrm{~mm}$. A detailed description of the calculations can be found elsewhere ${ }^{16}$. In addition to these calculations, we also counted a contact when the distance to the line segments of the planes was smaller than the target radius.

Subsequently, we determined whether a segment had made contact with the target during the trial. The percentage of trials in each condition where this happened was calculated for each segment. For the distal segments (fingertips) we also calculated at what moment in time the target was touched. The trial was divided into 20 intervals with a length of $5 \%$ of the reaction time. In each interval we determined whether a finger made contact with the target.

Movement classification. To classify the movements, four different strategies were defined: shuffle, grasp, manipulate and thumb. The set of criteria variables that was used to classify the movements is shown in Table 2: the median speed of the thumb and ring finger with respect to the hand, the volume of the convex hull the thumb positions enveloped and the number of turning points (from the angular velocity) of the ring finger.

All variables were normalised by dividing by the mean value over all conditions for each participant separately. The normalised variable set for each trial was matched to the pattern of high and low values for each strategy, as described in Table 2. The pattern of a strategy was represented as 0.7 for low values and 1.3 for high values $(30 \%$ below and above the mean). For example, the pattern of the thumb strategy would be $[1.3,0.7,1.3,0.7]$. For each strategy, the pattern was subtracted from the calculated values of the trial that was analysed. The sum of squares was calculated for these differences and the strategy with the smallest sum of squares was chosen for that trial. In this way, each trial was assigned one strategy. The automatic classification was not always similar to human observation (see also the Supplementary Information), but a human observer is also subjective and should not be seen as the standard.

Statistics. The statistics are described in the Results section. In all analyses, an $\alpha$-value of 0.05 was used. If Mauchley's test of sphericity was violated, a Greenhouse-Geisser correction was applied. Post-hoc tests were performed using paired $t$-tests. A Bonferroni correction was applied to relevant comparisons that were chosen in advance. Effect sizes are given as partial eta squared $\left(\eta_{p}^{2}\right)$.
1. Treisman, A. \& Souther, J. Search asymmetry: a diagnostic for preattentive processing of separable features. J. Exp. Psychol. Gen. 114, 285-310 (1985).

2. Lederman, S. J. \& Klatzky, R. L. Relative availability of surface and object properties during early haptic processing. J. Exp. Psychol. Human. 114, 1680-1707 (1997).

3. Plaisier, M. A., Bergmann Tiest, W. M. \& Kappers, A. M. L. Haptic pop-out in a hand sweep. Acta Psychol. 128, 368-377 (2008).

4. Plaisier, M. A., Bergmann Tiest, W. M. \& Kappers, A. M. L. Salient features in 3-D haptic shape perception. Atten. Percept. Psycho. 71, 421-430 (2009).

5. Plaisier, M. A. \& Kappers, A. M. L. Cold objects pop out! LNCS 6192, 219-224 (2010).

6. Van Polanen, V., Bergmann Tiest, W. M. \& Kappers, A. M. L. Haptic pop-out of movable stimuli. Atten. Percept. Psycho. 74, 204-215 (2012).

7. Van Polanen, V., Bergmann Tiest, W. M. \& Kappers, A. M. L. Haptic search for hard and soft spheres. PLoS ONE 7, e45298 (2012).

8. Zelinsky, G. J. \& Sheinberg, D. L. Eye movements during parallel-serial visual search. J. Exp. Psychol. Human. 23, 244-262 (1997).

9. Overvliet, K. E., Smeets, J. B. J. \& Brenner, E. Haptic search with finger movements: using more fingers does not necessarily reduce search times. Exp. Brain Res. 182, 427-434 (2007).

10. Smith, A. M., Gosselin, G. \& Houde, B. Deployment of fingertip forces in tactile exploration. Exp. Brain Res. 147, 209-218 (2002).

11. Van Polanen, V., Bergmann Tiest, W. M. \& Kappers, A. M. L. Movement strategies in a haptic search task. IEEE World Haptics Conference (WHC), 287-280 (2011)

12. Van Polanen, V., Bergmann Tiest, W. M. \& Kappers, A. M. L. Integration and disruption effects of shape and texture in haptic search. PLoS ONE 8, e70255 (2013).

13. Lederman, S. J. \& Klatzky, R. L. Hand movements - a window into haptic object recognition. Cognitive Psychol 19, 342-368 (1987).

14. Jansen, S. E. M., Bergmann Tiest Wouter, M. \& Kappers Astrid, M. L. Identifying haptic exploratory procedures by analyzing hand dynamics and contact force. IEEE Trans. Haptics 6, 464-472 (2013).

15. Johansson, R. S. \& Flanagan, J. R. Coding and use of tactile signals from the fingertips in object manipulation tasks. Nat. Rev. Neurosci. 10, 345-359 (2009).

16. Van Polanen, V. Findings in haptic (re) search $\mathrm{PhD}$ dissertation, VU University Amsterdam, Faculty of Human Movement Sciences (2014), http://dare.ubvu.vu. $\mathrm{nl} / \mathrm{handle} / 1871 / 51344$

17. Dahiya, R. S., Metta, G., Valle, M. \& Sandini, G. Tactile sensing - from humans to humanoids. IEEE Transactions on Robotics 26, 1-20 (2010).

18. Yousef, H., Boukallel, M. \& Althoefer, K. Tactile sensing for dexterous in-hand manipulation in roboticsA review. Sensors and Actuators A: physical 167, 171-187 (2011).

19. Coren, S. The left-hander syndrome: The causes and consequences of lefthandedness. (Vintage Books, New York, 1993)

\section{Acknowledgments}

This work was supported by the European Commission with the Collaborative Project no. 248587, "THE Hand Embodied", within the FP7-ICT-2009-4-2-1 program "Cognitive Systems and Robotics".

\section{Author contributions}

V.P., W.M.B.T. and A.M.L.K. designed the experiment. V.P. performed the experiment, analysed the data and prepared the figures. V.P., W.M.B.T. and A.M.L.K. wrote and reviewed the manuscript.

\section{Additional information}

Supplementary information accompanies this paper at http://www.nature.com/ scientificreports

Competing financial interests: The authors declare no competing financial interests.

How to cite this article: van Polanen, V., Bergmann Tiest, W.M. \& Kappers, A.M.L. Target contact and exploration strategies in haptic search. Sci. Rep. 4, 6254; DOI:10.1038/ srep06254 (2014)

This work is licensed under a Creative Commons Attribution-NonCommercialNoDerivs 4.0 International License. The images or other third party material in this article are included in the article's Creative Commons license, unless indicated otherwise in the credit line; if the material is not included under the Creative Commons license, users will need to obtain permission from the license holder in order to reproduce the material. To view a copy of this license, visit http:// creativecommons.org/licenses/by-nc-nd/4.0/ 